\title{
Statistical analysis of surface displacements - an example from the Åknes rockslide, western Norway
}

\author{
T. Nordvik ${ }^{1}$ and E. Nyrnes ${ }^{2}$ \\ ${ }^{1}$ Norwegian University of Science and Technology, Trondheim, Norway \\ ${ }^{2}$ StatoilHydro Research Centre, Trondheim, Norway
}

Received: 3 December 2008 - Revised: 29 April 2009 - Accepted: 7 May 2009 - Published: 14 May 2009

\begin{abstract}
Analyses of displacement measurements may provide valuable insight into the characteristics and behaviours of landslides. This paper demonstrates the application of statistical analysis to displacement data collected with Global Positioning System (GPS), total stations and extensometers at the Åknes rockslide site, western Norway. The Åknes rockslide has particular interest due to the potential for catastrophic consequences if the rockslide accelerates into a rock avalanche and hits the fjord below. This would generate a tsunami in the adjacent fjord system and pose a threat to local settlements and infrastructure as well as to the many tourists visiting nearby areas. The analyses reported in this paper pay special attention to the newly available time series obtained from seven permanent GPS stations. The results from these continuously monitored GPS stations are believed to be an important contribution to the understanding of the complex displacement pattern evident from previous investigations. Results from the statistical analyses show that the displacement rates can be modelled as linear trends superimposed with periodic (sinusoidal) components. This indicates constant average displacement rates with no persistent accelerations. The annual displacement rates estimated from GPS and extensometer measurements range from a few millimetres to about $8 \mathrm{~cm}$, whereas the periodical fluctuations typically have maximum amplitudes of 1-2 $\mathrm{mm}$. Some interpretations of the periodical fluctuations are presented. High correlations between displacements and the groundwater level, measured in a borehole at the upper part of the slope, are evident for extensometers located across the back scarp. For the GPS control points located further down the slope, this correlation is, however, not so clear.
\end{abstract}

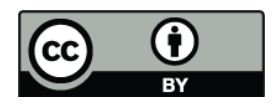

Correspondence to: T. Nordvik (trond.nordvik@ntnu.no)

\section{Introduction}

This paper focuses on statistical analysis of displacement data and demonstrates the application of statistical methods to rock slope displacement data collected at the Åknes rockslide site, western Norway. The Åknes rockslide is among the world's most investigated rockslides (Ganerød et al., 2008). This rockslide has particular interest due to the potential for catastrophic consequences if the rockslide accelerates into a rock avalanche and hits the fjord below. This would generate a tsunami in the adjacent fjord system and pose a threat to local settlements and infrastructure as well as to tourists onboard the many cruise ships visiting the fjord located below the unstable rock slope. The objective of this paper is not to give a comprehensive evaluation of all available displacement data at Åknes, but rather to emphasize on the gain from using statistical methods as part of the overall landslide assessment methodology. Displacement measurements are often evaluated without paying much attention to their statistical properties. For example, this is the case when only visual examinations of raw-data plots are used. As statistical methods explicitly account for the statistical properties of the data, they may provide a more objective basis for data analysis.

The following list contains some key problems which will be addressed by the statistical analyses;

- Identify moving and stable control points by detection of statistically significant displacements.

- Estimate the displacement rate with corresponding confidence interval (CI) for each control point.

- Establish and quantify periodic variations in displacement rates.

- Estimate correlations between displacement rates and the groundwater level.

Published by Copernicus Publications on behalf of the European Geosciences Union. 


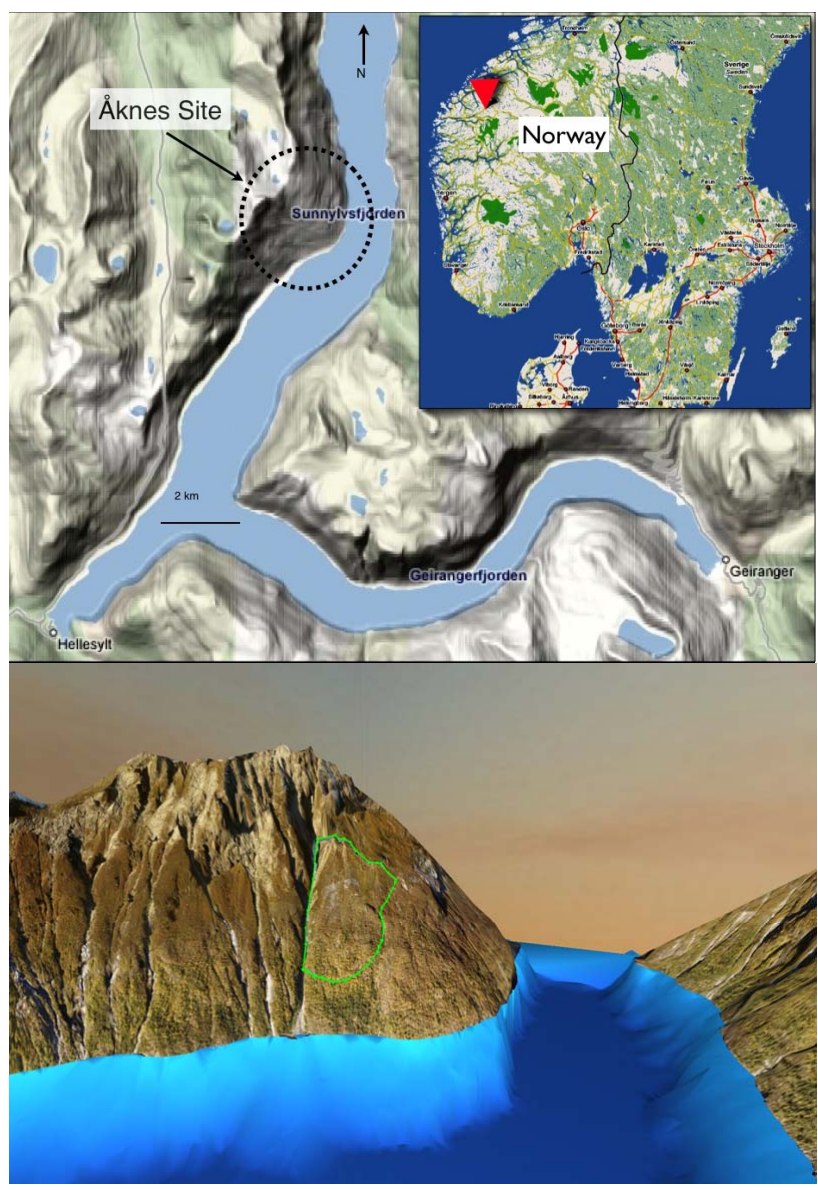

Fig. 1. Top: Location of the Åknes site. Bottom: A perspective view of the site. The boundary of the assumed unstable part of the slope is indicated with a green polygon.

- Identify control points showing similar displacement pattern.

- How to cope with imperfections in real world data, such as noise, offsets and missing observations as well as separating artefacts from actual displacements.

This paper starts with a brief description of displacement analysis in general. Then short introductions of the Åknes rockslide and the relevant displacement datasets are given. The core of this paper is the description of the methods used for analyzing displacement data from the Åknes rockslide along with the results obtained. Finally, some conclusions are drawn from the results.

\subsection{Displacement analysis}

Displacement or deformation analysis is an important tool for landslide assessment. The desired type of output from these analyses depends on a number of factors such as the objective of the analysis, the type of slide, the material in- volved (clay, rock etc.) as well as the spatiotemporal resolution and accuracy of the available measurements. Caspary et al. (1990) describe a general approach for deformation analysis which may also serve as basis for landslide analyses. Most approaches for deformation analysis are closely related to Least Square (LS) estimation using the GaussMarkov (GM) model which will be addressed in Sect. 2.2. In some applications the outcome from a deformation analysis is simply the identification of points showing significant displacement, in absolute position, between two or more epochs (e.g. Savšsek-Safi et al., 2006), whilst in other applications the final outcome may be the identification blocks within a sliding area (e.g. Haberler-Weber, 2005) or the estimation of model parameters concerning translation, shear, rotation and scale, also known as strain estimation (e.g. Tzenkov and Gospodinov, 2003; Teza et al., 2008). The estimated model parameters are subsequently used for interpretations (e.g. Tzenkov and Gospodinov, 2003) and predictions of a slide's progress (e.g. Petly et al., 2002). As one of the major issues in displacement analyses is to detect significant displacement of individual control points or significant network deformations, statistical analyses and particularly hypothesis testing are essential tools. For example, the global congruency test (e.g. Cooper, 1987; Kennie and Petrie, 1990; Denli and Deniz, 2003) may be a useful first step towards the examination of the total deformation of a network between two epochs. If the observed deformation is small compared to the accuracy of the measurements, the network is regarded as congruent at those two epochs, otherwise the observed deformation is deemed significant, which usually requires further analyses. This may be the estimation of kinematics parameters such as velocity and acceleration (e.g. Sitros et al., 2004; Brückl et al., 2006; Pytharouli et al., 2007).

If time series data are available, spectral analysis may be a useful technique to establish periodical variations such as seasonal variations (e.g. Pytharouli et al., 2007). The most widely used spectral analysis is the Fourier analysis of which the basics are outlined in Sect. 2.3.

\subsection{The Åknes rockslide}

Åknes is located in western Norway (Fig. 1). In 2004, an extensive mapping and monitoring program was initiated for the unstable rock slope at Åknes. This program is still ongoing and includes, amongst others, various types of surface displacement measurements which form the basis for the statistical analyses reported in this paper. Kveldsvik et al. (2006) give a geological evaluation of the early displacement measurements collected at Åknes. They concluded that the Åknes rockslide currently was in a steady creep phase and that an accelerating phase is expected prior to a possible catastrophic failure.

The upper boundary of the unstable area is clearly defined by an $800 \mathrm{~m}$ long scarp located about $800-900 \mathrm{~m}$ above sea level. However, the extent and volume of the unstable 


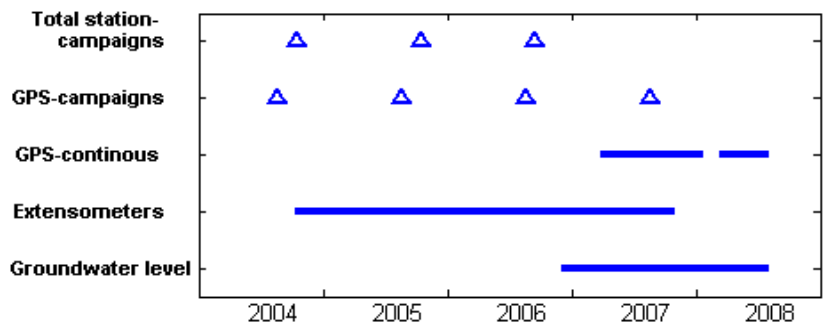

Fig. 2. Chronological overview of the datasets used in this paper.

rock mass, as well as the number and extents of the individual rock blocks involved, are not fully known. Nordvik et al. (2009) point out three different scenarios having volume estimates ranging from 20 to 85 million cubic metres. For a detailed description of the geological conditions at Åknes see Ganerød et al. (2008).

\subsection{Description of data}

Seven permanent GPS control points, three extensometer control points as well as the groundwater level were repeatedly measured for periods of time providing time series data. A number of total station control points and some additional GPS control points were only measured for a few epochs, denoted as survey campaigns in this paper, Fig. 2.

During a period of nearly 500 days, seven permanent GPS receivers have been operating at the Åknes rockslide site. Measurements were carried out as relative measurements using a GPS base station located at a fixed point close to the unstable rock slope. The locations of the control points and the base station together with the assumed boundaries of the unstable part of the rock slope are shown in Fig. 3.

The GPS antennas were mounted on top of about 3 meter tall aluminium pillars with cone-shaped caps to prevent snow and ice from interfering with the GPS signals. Six of the control points were located inside the suspected unstable part of the slope. The remaining control point (GPS 2) was located above the back scarp and thus assumed stable. During operation this control point was moved to a new location, a few metres away from the initial position, causing a shift in its time series.

The $\mathrm{x}-$, $\mathrm{y}$ - and $\mathrm{z}$-coordinates of the seven control points were recorded with a nominal sampling interval of $12 \mathrm{~h}$. However, due to various circumstances this constant sampling interval was not maintained for the entire time series. For example; during a period of nearly 40 days, the power supply was down due to damages caused by lightening. Consequently no measurements were recorded during this period, leaving major gaps in the time series. Moreover, some settings related to the atmospheric correction model used for processing were also altered during operation. This is evident as shifts in the time series as well as changes in the noise levels (Fig. 4).

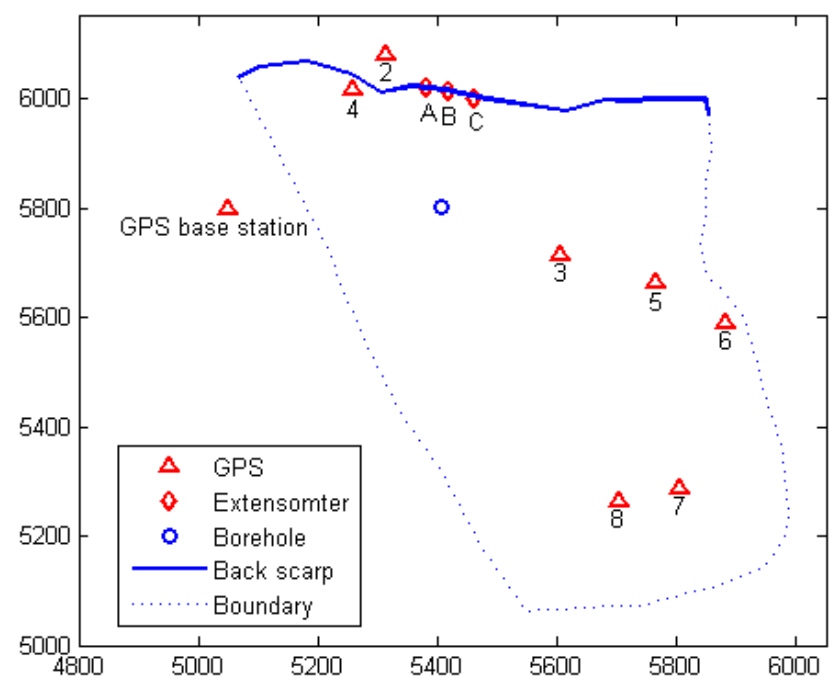

Fig. 3. Locations of permanent GPS and extensometer control points together with the assumed boundaries for the unstable part of the slope. (Coordinates are given in a local system; the transformation to UTM Zone $32 \mathrm{~N}$ is simply +390000 and +6890000 for the $\mathrm{x}$ - and $\mathrm{y}$-coordinate, respectively).

Data from three extensometers measuring the extension across the back scarp (Fig. 3) were included in this investigation. Even though the extensometer measurements were recorded with a nominal sampling interval of five minutes, only a single observation per day was considered for the analyses in this paper. An approximate orientation was measured for each extensometer. Assuming constant orientations of the extensometers, this allows for an approximate decomposition of the extensometer readings into changes in the $\mathrm{x}-, \mathrm{y}-$ and $\mathrm{z}-$ direction. This is convenient for comparing results from extensometer with those obtained from GPS and total stations. However, the extensometers in question are oriented in the North-South direction along the slope; therefore changes in the $\mathrm{x}$-components are not detectable.

As the groundwater level is assumed to have major influence on the displacement rates, daily groundwater records from a borehole located at the upper part of the slope (Fig. 3) were also considered for this investigation.

\section{Methods}

\subsection{Principal component analysis}

The Principal Components (PCs) are simply the eigenvectors of a (covariance) matrix. The eigenvector corresponding to the largest eigenvalue is denoted as the first principal component (PC1), the eigenvector corresponding to the second largest eigenvalue is denoted as the second principal component (PC2) and so on. Principal components depend solely on the covariance matrix of the data, and thus, their 

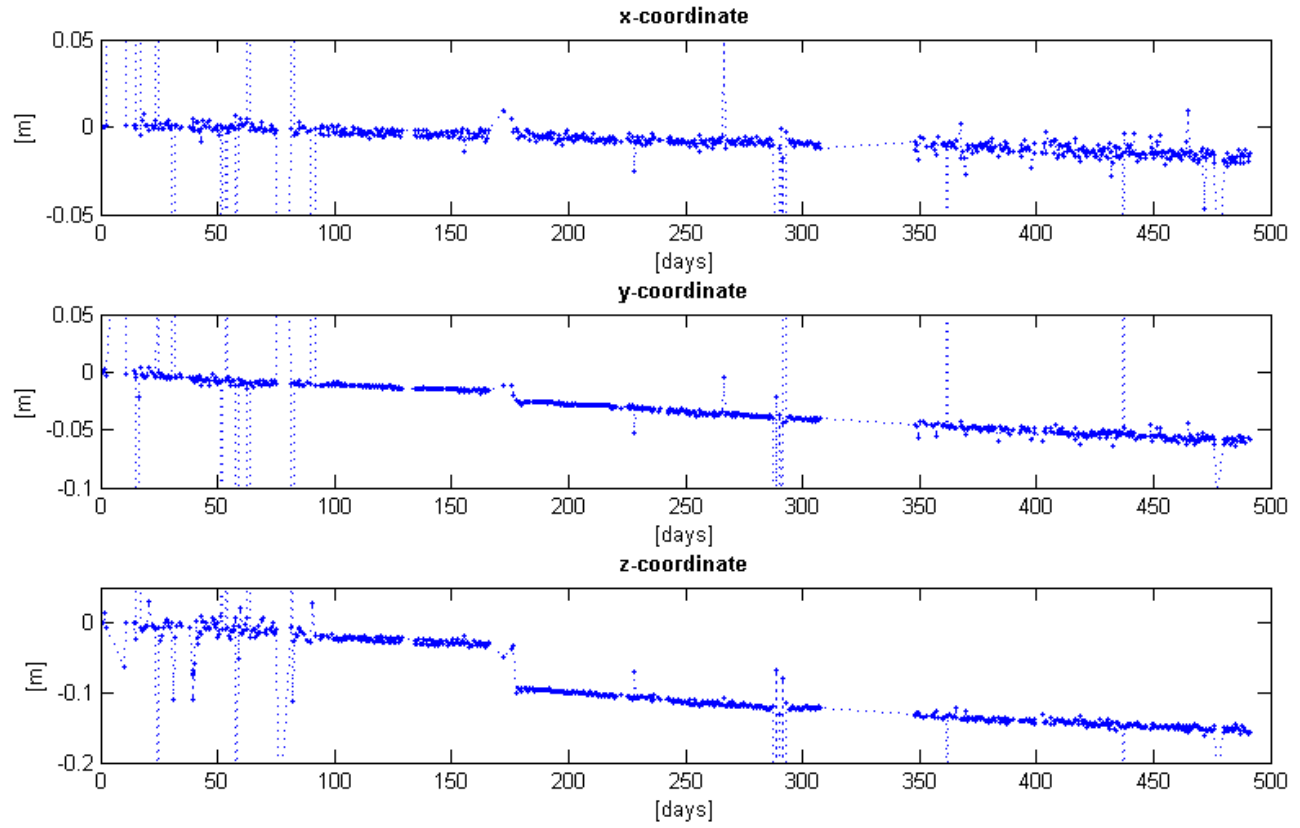

Fig. 4. Plots of raw GPS time series for point 4. (Day 0 corresponds to 26 March 2007).

development does not require a multivariate normal assumption (Johnson and Wichern, 2002). In this investigation Principal Component Analysis (PCA) is solely used for interpretation but PCA is also commonly used for data reduction purposes. The reader is referred to Johnson and Wichern (2002) and Davis (2002) for a more detailed description of PCA.

\subsection{Trend estimation from time series}

As a first step towards extracting the useful information from the available time series, trend models were fitted to each individual time series. This subsection outlines the chosen procedures and principles used for the trend modelling.

Each GPS control point has three corresponding time series; one for each of the $\mathrm{x}$-, $\mathrm{y}$ - and $\mathrm{z}$-coordinates. The recorded coordinates are given with respect to the UTM system. In order to get a more appropriate scale for the observations, the first observation from each series was simply subtracted from the subsequent observations to obtain $\Delta x$, $\Delta y$ and $\Delta z$. Using delta values, as opposed to UTM coordinates, this eases human readability and is also preferable with respect to numerical stability.

Plots of the time series suggest linear trends (Fig. 4). However, there are a number of shifts which have to be considered as well as a number of gross errors, particularly at the first parts of the time series (Fig. 4). In order to calculate the magnitudes of the potential offsets, along with their significance levels, the models were extended with offset parameters which were estimated simultaneously with the other regression parameters. The estimated offsets for each of the $\mathrm{x}, \mathrm{y}, \mathrm{z}$ time series were corrected before the corresponding absolute displacement (distance) time series were calculated.

To eliminate the major portion of gross errors, all delta values exceeding a discretionally chosen threshold were removed before the actual trend estimation.

In this paper, the parameter estimation is based on the GM model (e.g. Koch, 1999), where the expectation of the observations is described as linear combinations of unknown parameters. The GM model is defined by Eq. (1):

$E(\boldsymbol{y})=\mathbf{X} \beta=\boldsymbol{y}+\boldsymbol{e}$ with $\operatorname{Cov}(\boldsymbol{y})=\operatorname{Cov}(\boldsymbol{e})=\sigma^{2} \boldsymbol{Q}_{e e}=\boldsymbol{C}_{e e}$

where $\boldsymbol{y}$ is a vector of observations, $\boldsymbol{\beta}$ is a vector of unknown parameters, $\mathbf{X}$ is a known design matrix, $\boldsymbol{Q}_{e e}$ is the co-factor matrix of the errors $\boldsymbol{e}$ and $\sigma^{2}$ is the variance of unit weight, usually unknown. In this paper the errors $\boldsymbol{e}$ are assumed to be normally distributed; $\boldsymbol{e} \sim N\left(\mathbf{0}, \sigma^{2} \boldsymbol{Q}_{e e}\right)$. The product of $\sigma^{2}$ and $\boldsymbol{Q}_{e e}$ is the error covariance matrix $\mathbf{C}_{e e}$, and the inverse of $\boldsymbol{Q}_{e e}$ is the weight matrix; $\mathbf{P}=\boldsymbol{Q}_{e e}^{-1}$.

Assuming a GM model containing different regression parameters such as constant term, linear trend and offsets, the structure of the design matrix $\mathbf{X}$ is:

$\boldsymbol{X}=\left[\begin{array}{ccccc}1 & t_{1} & 0 & \cdots & 0 \\ 1 & t_{2} & 0 & \cdots & 0 \\ \vdots & \vdots & \vdots & \vdots & \vdots \\ 1 & t_{k} & 1 & \cdots & 0 \\ 1 & t_{k+1} & 1 & \cdots & 0 \\ \vdots & \vdots & \vdots & \vdots & \vdots \\ 1 & t_{n-1} & 1 & \cdots & 1 \\ 1 & t_{n} & 1 & \cdots & 1\end{array}\right]$ and $\boldsymbol{\beta}=\left[\begin{array}{c}\beta_{1} \\ \beta_{2} \\ \vdots \\ \beta_{u}\end{array}\right], \boldsymbol{y}=\left[\begin{array}{c}y_{1} \\ y_{2} \\ \vdots \\ y_{n}\end{array}\right]$ 
where the first and the second column of $\mathbf{X}$ represent the constant term and linear trend respectively. Offset terms were introduced to model sudden unexpected shifts in the time series, and are represented by the digit one in the consecutive columns of $\mathbf{X}$. If no covariance information exists on the observations $\boldsymbol{y}$, the weight matrix $\mathbf{P}$ is diagonal. Further, if the variances are equal, the weight matrix $\mathbf{P}$ equals the identity matrix. The least squares estimates $\hat{\boldsymbol{\beta}}$ of the unknown parameters $\boldsymbol{\beta}$ are given by:

$\hat{\boldsymbol{\beta}}=\left(\mathbf{X}^{\mathbf{T}} \mathbf{P X}\right)^{-1} \mathbf{X}^{\mathbf{T}} \mathbf{P} \boldsymbol{y}$ with $\operatorname{Cov}(\hat{\boldsymbol{\beta}})=\hat{\sigma}^{2}\left(\mathbf{X}^{\mathbf{T}} \mathbf{P X}\right)^{-1}$

$=\hat{\sigma}^{2} \mathbf{Q}_{\hat{\beta} \hat{\beta}}=\mathbf{C}_{\hat{\beta} \hat{\beta}}$

where $\hat{\sigma}^{2}$ is the unbiased estimator of the variance $\sigma^{2}$ of unit weight obtained from:

$\hat{\sigma}^{2}=\frac{\hat{\boldsymbol{e}}^{T} \mathbf{P} \hat{\boldsymbol{e}}}{n-u}$

where the quadratic form $\hat{\boldsymbol{e}}^{T} \mathbf{P} \hat{\boldsymbol{e}}$ is the residual sum of squares and $n-u$ is the degrees of freedom, i.e. the difference between the number of observations in $\boldsymbol{y}$ and the number of unknowns in $\boldsymbol{\beta}$. The least squares estimates $\hat{\boldsymbol{e}}$ of the errors $\boldsymbol{e}$ are the residuals, and can be obtained from Eq. (5):

$\hat{\boldsymbol{e}}=\mathbf{X} \hat{\boldsymbol{\beta}}-\boldsymbol{y}$ with $\operatorname{Cov}(\hat{\boldsymbol{e}})=\hat{\sigma}^{2}\left[\boldsymbol{Q}_{e e}-\mathbf{X}\left(\mathbf{X}^{T} \mathbf{P X}\right)^{-1} \mathbf{X}^{T}\right]$

$=\hat{\sigma}^{2} \boldsymbol{Q}_{\hat{e} \hat{e}}$

After the first coarse approach for removal of obvious blunders, a certain amount of potential gross errors remained. The process of removing the remaining gross errors was carried out by analysing the standardized residuals. A standardized residual $\hat{d}_{i}$ is defined by:

$\hat{d}_{i}=\frac{\hat{e}_{i}}{\hat{\sigma}}$

that is, each residual $\hat{e}_{i}$ is divided by the estimated standard deviation of unit weight. The standardized residuals have zero mean and approximately unit variance (Montgomery et al., 2001). All observations corresponding to a standardized residual greater than 3.0 were considered as gross errors and removed from the dataset.

The hypotheses for testing the significance of the $j$ th parameter $\beta_{j}$ can be expressed as:

$H_{0}: \beta_{j}=0$ versus $H_{1}: \beta_{j} \neq 0, j \in\{0,1, \ldots, u-1\}$

The test statistic for testing the above hypotheses is given by:

$t_{0}=\frac{\hat{\beta}_{j}}{\sqrt{\boldsymbol{C}_{j j}}}$ where $\boldsymbol{C}_{j j}$ is the variance of $\hat{\beta}_{j}$, i.e. the $j$ th diagonal element in the covariance matrix $\boldsymbol{C}_{\hat{\boldsymbol{\beta}} \hat{\beta}}$. The null hypothesis $H_{0}$ is rejected if:

$\left|t_{0}\right|>t_{\alpha / 2, n-u-1}$

where $\alpha$ is the significance level of the test and $t_{\alpha} / 2, n-u-1$ is the upper $\alpha / 2$ quantile of a Student'st-distribution with $n-u-1$ degrees of freedom. A rejection of $H_{0}$ implies that the actual parameter $\beta_{j}$ is significantly different from zero, and should therefore be included in the model. A significance level $\alpha$ of 5\% is used for all hypotheses testing in this paper.

Processing one time series at a time, a complete model containing all possible regression parameters was first fitted to the time series. Then the parameter yielding the smallest absolute value of test statistic in Eq. (8) was chosen for deletion and a new least squares estimation was performed. The same procedure was repeated until only significant parameters remained. This parameter selection approach is known as Backward Elimination (e.g. Miller, 2002).

\subsection{Spectral analysis}

The residuals obtained from the final trend models were further investigated by spectral analysis to detect possible periodic components in the time series. The most common spectral decomposition technique is the Fourier transform which enables the decomposition of any periodic function into a set of cosine and sine components that represent the frequency spectre. In order to apply Fourier analysis, the data should be equidistant. This implies that there should be no gaps within the time series. For the time series in question, this requirement was not met as the there were subintervals with missing observations. Moreover, removal of gross errors also caused additional gaps in the time series which had to be corrected. The initial residuals were therefore interpolated to obtain equidistant data. However, no interpolations were carried out for the "missing residuals" within the longer gaps ( $>10$ days); these were simply set to zero.

Using the discrete Fourier transform, a time series $y(t)$ can be represented as in Eq. (10) (Wei, 1990).

$$
\begin{aligned}
& y(t)=\sum_{k=0}^{n / 2}\left[a_{k} \cos (2 \pi k t / n)+b_{k} \sin (2 \pi k t / n)\right], \\
& t=1,2, \ldots, n
\end{aligned}
$$

Equation (10) can be set up as a GM model (Sect. 2.2) and the LS method can be used for estimation of the Fourier coefficients $a_{k}$ and $b_{k}$. A model containing only a single wave component with frequency $\omega_{1}$ has the following structure:

$\mathbf{X}=\left[\begin{array}{cc}\cos \left(\omega_{1} t_{1}\right) & \sin \left(\omega_{1} t_{1}\right) \\ \cos \left(\omega_{1} t_{2}\right) & \sin \left(\omega_{1} t_{2}\right) \\ \vdots & \vdots \\ \cos \left(\omega_{1} t_{n}\right) & \sin \left(\omega_{1} t_{n}\right)\end{array}\right] \quad \boldsymbol{\beta}=\left[\begin{array}{l}a_{1} \\ b_{1}\end{array}\right], \quad \boldsymbol{y}=\left[\begin{array}{c}y_{1} \\ y_{2} \\ \vdots \\ y_{n}\end{array}\right]$ 


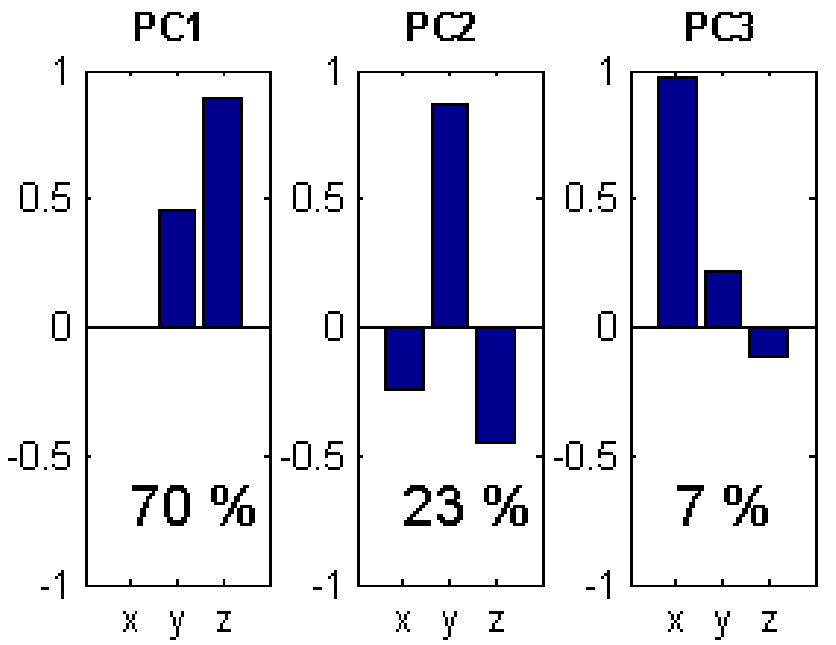

Fig. 5. Coefficients of the Principal Components (PCs) calculated from displacement vectors derived from GPS and total station surveys (cf. Figs. 6 and 7). The $\mathrm{x}$-coefficient of $\mathrm{PC} 1$ is zero; this means that $\mathrm{PC} 1$ lies in the yz-plane. The percentage associated with each $\mathrm{PC}$ is the portion of the total variability explained by this PC.

Each new Fourier frequency introduced adds two new columns to the design matrix $\mathbf{X}$; one cosine term and one sine term as well as the two corresponding Fourier coefficients to the parameter vector $\beta$. The $\boldsymbol{y}$ vector contains residuals from a trend model. If only the Fourier frequencies are used, the estimated coefficients $\hat{a}_{k}$ and $\hat{b}_{k}$ will be uncorrelated as the Fourier frequencies form an orthogonal basis. The first Fourier frequency is obtained from $\omega_{1}=2 \pi / T$, where $T$ denotes the total time span of the time series. For a unit sampling frequency, $T$ equals $n$. Consecutive Fourier frequencies are obtained by $\omega_{k}=k \omega_{1}$, where $k=2,3, \ldots, n / 2$, and their amplitudes are obtained from:

$\hat{A}_{k}=\sqrt{\hat{a}_{k}^{2}+\hat{b}_{k}^{2}}$

The significance of a single wave component $k$ is evaluated by means of its contribution to the weighted sum of squares. Relevant hypotheses become:

$H_{0}: a_{k}=b_{k}=0$ versus $H_{1}: a_{k} \neq 0 \vee b_{k} \neq 0$

The null hypothesis $H_{0}$ is rejected if:

$F=\frac{\left(\boldsymbol{y}^{T} \mathbf{P} \boldsymbol{y}-\hat{\boldsymbol{e}}^{T} \mathbf{P} \hat{\boldsymbol{e}}\right) / 2}{\left(\hat{\boldsymbol{e}}^{T} \mathbf{P} \hat{\boldsymbol{e}}\right) /(n-2)}>F_{(2, n-2, \alpha)}$,

that is, if the test statistic $F$ exceeds the upper $\alpha$-percentage point of a Fisher distribution with 2 and $n-2$ degrees of freedom. In case of equal variance and uncorrelated observations, the weight matrix $\mathbf{P}$ becomes the identity matrix which can be omitted from Eq. (14). The same test statistics (Eq. 14) can also be used for testing the simultaneous significance of a set of wave components, providing that the degrees of freedom are updated accordingly. However, in this paper only one wave component was reported for each time series; the one that gave the largest value of the test statistic in Eq. (14).

\subsection{Testing for equality of displacement vectors}

A relevant question with respect to displacement analyses is whether two or more vectors are equal, i.e. whether the estimates $\hat{\boldsymbol{x}}_{i}$ and $\hat{\boldsymbol{x}}_{j}$ of two different displacement vectors have the same expectation value:

$H_{0}: E\left(\hat{\boldsymbol{x}}_{i}\right)=E\left(\hat{\boldsymbol{x}}_{j}\right)$ versus $H_{1}: E\left(\hat{\boldsymbol{x}}_{i}\right) \neq E\left(\hat{\boldsymbol{x}}_{j}\right)$

Under the assumption of normally distributed and uncorrelated displacement vectors;

$\hat{\boldsymbol{x}}_{i} \sim N\left(\boldsymbol{\mu}_{i}, \boldsymbol{C}_{i}\right)$ and $\hat{\boldsymbol{x}}_{j} \sim N\left(\boldsymbol{\mu}_{j}, \boldsymbol{C}_{j}\right)$ with $\operatorname{Cov}\left(\hat{\boldsymbol{x}}_{i}, \hat{\boldsymbol{x}}_{j}\right)=\mathbf{0}$,

the null hypothesis $H_{0}$ can be evaluated by means of a chisquared distributed random variable. The null hypothesis is rejected if:

$T^{2}=\left(\hat{\boldsymbol{x}}_{i}-\hat{\boldsymbol{x}}_{j}\right)^{T}\left(\boldsymbol{C}_{i}+\boldsymbol{C}_{j}\right)^{-1}\left(\hat{\boldsymbol{x}}_{i}-\hat{\boldsymbol{x}}_{j}\right)>\chi_{k, \alpha}^{2}$

where $\chi_{k, \alpha}^{2}$ is the upper $\alpha$ quantile of the chi-squared distribution with $k$ degrees of freedom. In this case $k$ equals 3 , i.e. the dimension of the displacement vectors.

\subsection{Cross-correlation}

The cross-covariance between two jointly stationary stochastic processes $x_{t}$ and $y_{t}$ with lag parameter $k$ is defined by (Wei, 1990):

$\gamma_{x y}(k)=E\left[\left(x_{t}-\mu_{x}\right)\left(y_{t+k}-\mu_{y}\right)\right]$,

$k \in\{0, \pm 1, \pm 2, \ldots$.$\} ,$

where the index $t$ refers to an observation made at time $t$. For large de-trended samples of size $n$ with constant variance, the estimated cross-covariance is obtained from:

$\hat{\gamma}_{x y}(k)=\frac{1}{n} \sum_{t=0}^{n-k-1} x_{t} y_{t+k}$

The conversion from cross-covariance to cross-correlation involves the normalization;

$\hat{\rho}_{x y}(k)=\frac{\hat{\gamma}_{x y}(k)}{\hat{\sigma}_{x} \hat{\sigma}_{y}}$,

where $\hat{\sigma}_{x}$ and $\hat{\sigma}_{y}$ are the estimated standard deviations of $x_{t}$ and $y_{t}$. 

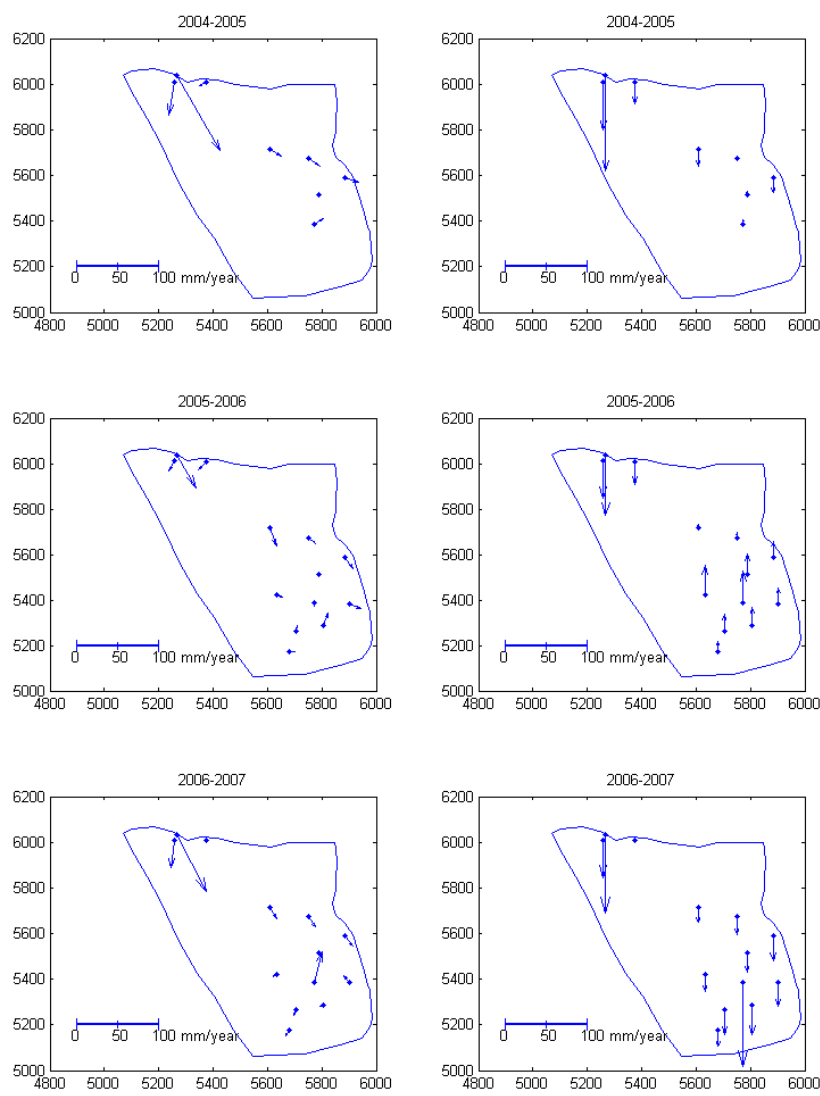

Fig. 6. Displacement vectors derived from GPS survey campaigns. Left column: horizontal displacements. Right column: vertical displacements.

\section{Results}

A principal component analysis was carried out for displacement vectors derived from GPS and total station survey campaigns (Fig. 5). The principal components are simply a rotation (orthogonal transformation) of the three initial coordinate axes such that the first principal component (PC1) represents the direction of most variability in the displacements. PC1 roughly corresponds to the down slope direction which is sensible since the largest displacement vectors go in this direction together with the fact that some of the control points show displacements close to zero. PC2 indicates the direction orthogonal to PC1 showing the second largest displacement variability. The direction of $\mathrm{PC} 2$ roughly corresponds to the slope's normal vector. The direction of PC3, orthogonal both to $\mathrm{PC} 1$ and $\mathrm{PC} 2$, roughly corresponds to the across slope direction. That is, there is the least variability in displacement across the slope.

Figures 6 and 7 show displacement vectors derived from GPS and total station survey campaigns carried out in 2004, 2005, 2006 and 2007. These vectors were obtained by taking the differences between LS adjusted coordinates at two consecutive epochs.
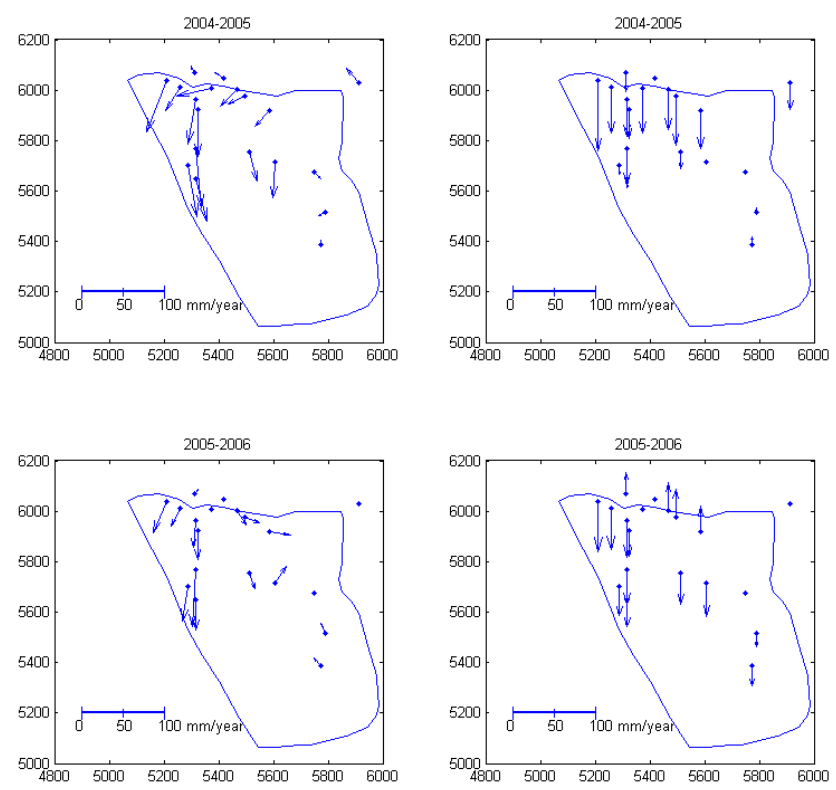

Fig. 7. Displacement vectors derived from total station survey campaigns. Left column: horizontal displacements. Right column: vertical displacements.

An example of fitted trend models is shown in Fig. 8. Figure 9 shows displacement-vectors derived from time series (comparable to those shown in Figs. 6 and 7). The estimated annual displacements and the most significant periodic components are listed in Tables 1 and 2. The periodic components corresponding to the middle time series in Fig. 8, i.e. for the y-coordinate, are shown in Figs. 10 and 11.

Spectral analysis of the time series for the groundwater level shows that the most significant period is 378 days with corresponding amplitude of $1.62 \mathrm{~m}$.

From Fig. 9 the question arises whether the displacements vectors for point 3,5 and 6 are equal, meaning that the observed deviations in directions and magnitude are only caused by random errors. This question can be summarised by the following hypotheses;

$H_{0}: E\left(\hat{\boldsymbol{x}}_{3}\right)=E\left(\hat{\boldsymbol{x}}_{5}\right)=E\left(\hat{\boldsymbol{x}}_{6}\right)$ versus $H_{1}:$

$E\left(\hat{\boldsymbol{x}}_{3}\right) \neq E\left(\hat{\boldsymbol{x}}_{5}\right) \vee E\left(\hat{\boldsymbol{x}}_{3}\right) \neq E\left(\hat{\boldsymbol{x}}_{6}\right) \vee E\left(\hat{\boldsymbol{x}}_{5}\right) \neq E\left(\hat{\boldsymbol{x}}_{6}\right)$

These hypotheses can be tested by splitting into vector pairs. Test statistics from Eq. (17), for each feasible vector pair, are shown in Table 3. The null hypothesis $H_{0}$ is rejected if at least one individual test statistics exceeds the critical value. Table 3 shows that all individual test statistics far exceed the critical value. Consequently, $H_{0}$ was rejected.

A cross correlation analysis of displacements and the groundwater level in a borehole located in the upper part of the unstable slope was carried out. All datasets were detrended before the cross correlations were calculated. The results indicate strong correlations $\left(\left|\rho_{x y}\right|>0.7\right)$ between the 
Table 1. Absolute displacements (distance). Linear trends and most significant periodic components from GPS and extensometer time series.

\begin{tabular}{lllll}
\hline Point & Trend [mm/year] & $95 \%$ CI for trend [mm/year] & Period [days] & Amplitude [mm] \\
\hline GPS 2 & 2.8 & {$[2.3,3.2]$} & 162 & 0.8 \\
GPS 3 & 30.6 & {$[29.6,31.5]$} & 66 & 0.8 \\
GPS 4 & 81.4 & {$[80.5,82.4]$} & 134 & 1.6 \\
GPS 5 & 17.6 & {$[17.0,18.2]$} & 6 & 0.6 \\
GPS 6 & 25.8 & {$[25.2,26.5]$} & 22 & 0.8 \\
GPS 7 & 14.7 & {$[13.3,16.2]$} & 333 & 1.6 \\
GPS 8 & 4.9 & {$[3.8,6.0]$} & 46 & 1.3 \\
Ext A & 13.3 & {$[13.2,13.4]$} & $>$ & $>$ \\
Ext B & 23.0 & {$[22.9,23.1]$} & $>$ & $>$ \\
Ext C & 15.3 & {$[15.3,15.4]$} & 355 & 0.7 \\
\hline
\end{tabular}

>indicates long periods which can not be reliably established from the current time series.
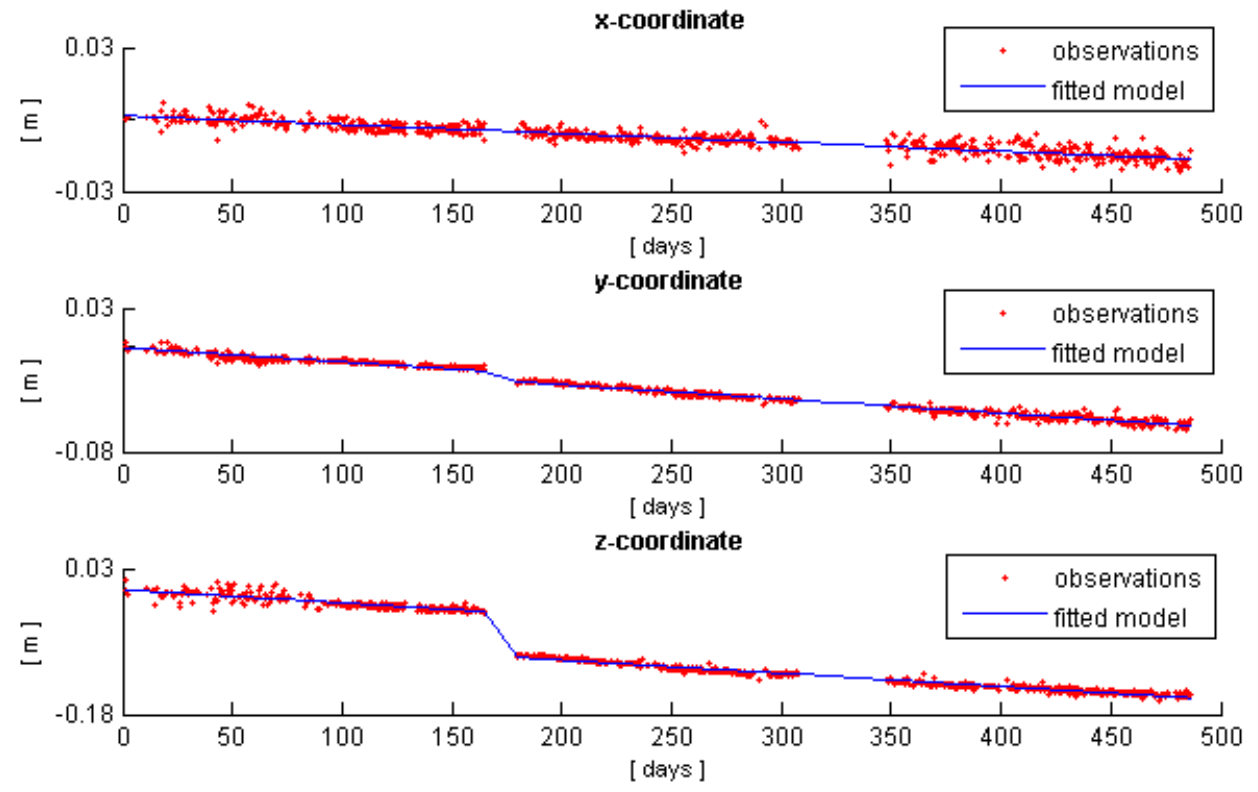

Fig. 8. Example of trend models. Linear trend models containing offset terms fitted to the time series of GPS control point 4 (correspond to the raw observations in Fig. 4).

groundwater level and the displacements measured by the extensometers at the back scarp (Table 4, Fig. 12). Increase in displacements for extensometer $\mathrm{A}$ and $\mathrm{B}$ tends to occur about three weeks after increase in groundwater level. For extensometer $\mathrm{C}$ the situation is, however, different as the maximum absolute correlation appears at lag zero. The correlation for extensometer $\mathrm{C}$ is negative which indicates that the displacement rate tends to slow down as the groundwater level increases. There are no strong correlations between groundwater level and displacements for the GPS control points (Table 4).

\section{Discussion}

The PCA carried out here is regarded as part of the exploratory analysis to gain overview of the situation. Not surprisingly, most of the variability is related to the down slope movement and subsidence of the slide (PC1 and PC2). The portion explained by PC3 is probably mostly due to observational noise.

As the GPS data were sampled with at least $12 \mathrm{~h}$ time spacing, the autocorrelation was neglected for these observations. The offsets detected from the GPS time series might correspond to minor failures in the slope but this is considered as highly unlikely as the same offset features are present for most of the GPS control points, but are not detectable from the extensometer time series. 
Table 2. Linear trends and most significant periodic components in $\mathrm{x}-\mathrm{y}$ - and $\mathrm{z}$-direction from GPS time series.

\begin{tabular}{lcllll}
\hline Point & Coordinate & Trend [mm/year] & $95 \%$ CI for trend [mm/year] & Period [days] & Amplitude [mm] \\
\hline \multirow{3}{*}{ GPS 2 } & $\mathrm{x}$ & - & - & $>$ & $>$ \\
& $\mathrm{y}$ & -2.8 & {$[-4.0,-1.8]$} & 158 & 0.8 \\
& $\mathrm{z}$ & - & - & 118 & 2.5 \\
GPS 3 & $\mathrm{x}$ & 12.8 & {$[12.3,13.3]$} & 118 & 0.7 \\
& $\mathrm{y}$ & -23.5 & {$[-24.2,-23.0]$} & 172 & 0.4 \\
& $\mathrm{z}$ & -14.2 & {$[-15.9,-12.5]$} & 62 & 1.1 \\
GPS 4 & $\mathrm{x}$ & -13.5 & {$[-13.9,-13.1]$} & 29 & 0.5 \\
& $\mathrm{y}$ & -39.9 & {$[-40.5,-39.2]$} & 339 & 0.8 \\
& $\mathrm{z}$ & -69.3 & {$[-70.7,-67.8]$} & 134 & 1.7 \\
GPS 5 & $\mathrm{x}$ & 11.2 & {$[10.4,12.1]$} & 127 & 0.8 \\
& $\mathrm{y}$ & -9.4 & {$[-10.0,-8.7]$} & 55 & 0.4 \\
& $\mathrm{z}$ & -9.8 & {$[-11.3,-8.3]$} & 23 & 1.1 \\
GPS 6 & $\mathrm{x}$ & 16.2 & {$[14.0,18.5]$} & 120 & 0.7 \\
& $\mathrm{y}$ & -17.9 & {$[-19.6,-16.3]$} & 176 & 0.8 \\
& $\mathrm{z}$ & -7.7 & {$[-9.7,-5.7]$} & 23 & 1.8 \\
GPS 7 & $\mathrm{x}$ & -10.9 & {$[-13.8,-8.1]$} & 282 & 1.2 \\
& $\mathrm{y}$ & - & - & 159 & 0.6 \\
& $\mathrm{z}$ & 8.9 & {$[2.9,14.9]$} & 16 & 1.8 \\
GPS 8 & $\mathrm{x}$ & 4.6 & {$[3.0,6.2]$} & 62 & 1.2 \\
& $\mathrm{y}$ & 1.7 & {$[0.9,2.6]$} & 203 & 0.6 \\
& $\mathrm{z}$ & - & - & 147 & 2.1 \\
\hline
\end{tabular}

- indicates non-significant values.

$>$ indicates long periods which can not be reliably established from the current time series.
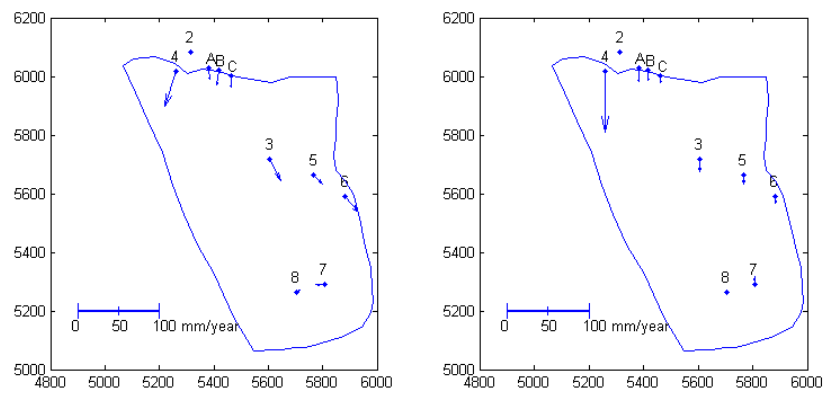

Fig. 9. Displacement vectors derived from GPS and extensometer time series. Left: horizontal displacements. Right: vertical displacements.

From the GPS time series, trends were estimated both for the absolute displacements (i.e. when only distances were considered) and for the individual coordinates $\mathrm{x}, \mathrm{y}$ and $\mathrm{z}$. All continuously monitored control points show significant displacements for at least one of their coordinates. This applies even to control point 2 which was assumed stable. Even though the estimated annual displacement for control point 2 is less than $3 \mathrm{~mm}$, this calls for further monitoring.

The estimated periodic components for the y-coordinate of GPS 4 (339 days, Table 2) as well as for Ext C (355 days, Table 1) are likely caused by changes in the groundwater level

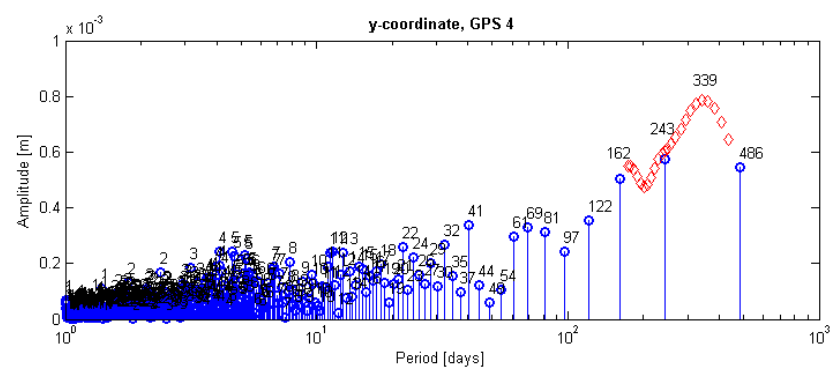

Fig. 10. Periodogram for the y-coordinate of GPS 4. Each vertical line represents a Fourier frequency with the corresponding wavelength indicated above. To obtain the most significant frequency for this time series, the Fourier frequency having the largest amplitude (wavelength 243) was used as a starting point. Repeated calculations, using only one periodic component, were then carried out until an approximate optimum frequency was reached (wavelength 339, cf. Fig. 11).

as the most significant periodic component for the groundwater level is also close to one year. Some of the time series show periodic components close to six months e.g. the y-coordinates of GPS 3 and GPS 6 (172 and 176 days respectively, Table 2) which indicate bi-annual variations. This may be related to snowmelt in the spring and increased precipitation in the autumn. Moreover, the time series for the y-coordinates of GPS 2, GPS 7 and GPS $8(158,159$ and 
Table 3. Test statistics regarding equality of displacement vectors.

\begin{tabular}{ccc}
\hline Vector combination $\boldsymbol{i}, \boldsymbol{j}$ & Test statistic $\left(T_{\boldsymbol{i}, \boldsymbol{j}}^{2}\right)$ & $T_{\boldsymbol{i}, \boldsymbol{j}}^{2}>\boldsymbol{\chi}_{3,0.05}^{2}=7.81$ \\
\hline 3,5 & 1026 & Yes \\
3,6 & 72 & Yes \\
5,6 & 109 & Yes \\
\hline
\end{tabular}

Table 4. Cross-correlations between groundwater level and absolute displacements.

\begin{tabular}{lrr}
\hline Control point & $\rho_{x y}[-1,1]$ & Lag [days] \\
\hline GPS 2 & 0.32 & -54 \\
GPS 3 & 0.20 & -45 \\
GPS 4 & -0.22 & 9 \\
GPS 5 & 0.18 & -42 \\
GPS 6 & 0.20 & -51 \\
GPS 7 & 0.29 & -16 \\
GPS 8 & -0.27 & 48 \\
Ext A & 0.73 & 21 \\
Ext B & 0.71 & 22 \\
Ext C & -0.78 & 0 \\
\hline
\end{tabular}

203 days respectively, Table 2) may also be related to this biannual variation but their estimated periods may be affected both by noise in the observations as well as the physical conditions of the rocks lope.

A well known problem when dealing with real world time series is that the total time span is too short to prove the existence of the longer periods. This applies to some of the time series analyzed here (e.g. Ext A and Ext B, Table 1). For example, Pytharouli et al. (2007) established the existence of periods between 4 and 7.5 years for two landslides in Greece. Moreover, it can be considered as a trade off whether to use the "fixed set" of Fourier frequencies which are uncorrelated but indirectly determined by the total length of the time series, or to use an arbitrary (probing) frequency being the optimal. If any arbitrary frequency is used, this implies some limitations with respect to interpretation of consecutive frequency estimates as these will no longer be uncorrelated. If the frequencies in question correspond to long wavelengths, with respect to the total time span, the approximation errors introduced by using Fourier frequencies are generally more notable. Therefore, in this paper only the single optimal frequencies, in terms of highest test statistic in Eq. (14), were listed. However, this implies that remaining periodical components are omitted even though they may be statistically significant. All periodical components reported in this paper were statistically significant which gives good indications that these periods are realistic, even though some of them are long compare to the total time span of the time series.

An important issue associated with the spectral analysis is

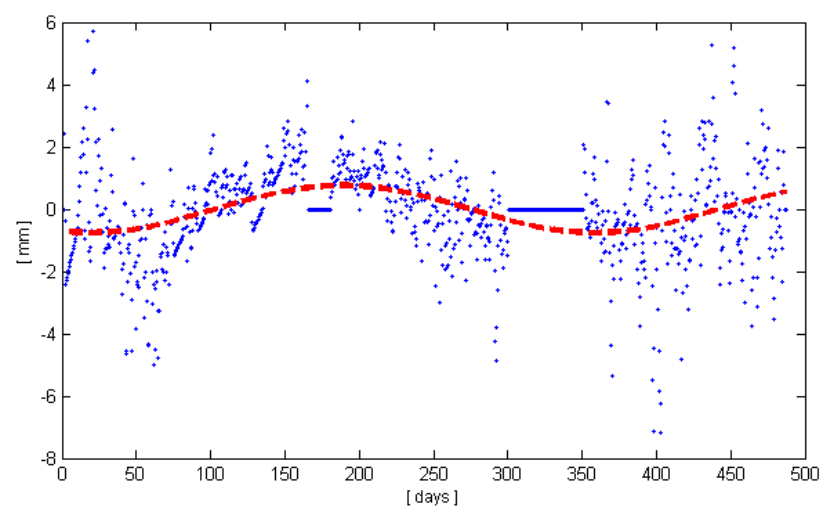

Fig. 11. Periodic fluctuations. The most significant wave component is fitted to the y-coordinate of GPS 4. The period is 339 days and the amplitude is approximately $0.8 \mathrm{~mm}$. The peak appears in early autumn as day 0 corresponds to 26 March 2007. (Apparent artefacts stem from interpolation and insertion of "zero-residuals" to get equidistant data).
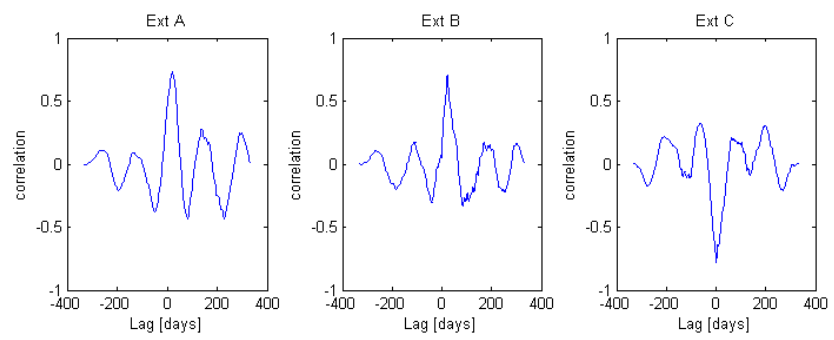

Fig. 12. Cross-correlations between groundwater level in borehole and displacements for extensometer A, B and C.

to separate the periodical components related to un-modelled effects from those related to the actual displacement of the rock slope. As the GPS measurements were calculated as relative measurements i.e. as vectors from a fixed base station, one should not expect to find the typical periodical components as for absolute GPS measurements such as ocean loading, Earth tide and the Chandler wobble. However, there may be other periodical components that can affect the time series but still not related to the physical motion of the rock slope. For example, the heat expansion of the aluminium pillars, used as base for the GPS antennas, will be in the order of magnitude $2 \mathrm{~mm}$ from one extreme to the other assuming a variation in temperature of 30 degrees Celsius (thermal expansion coefficient for Aluminium is $2.3 \cdot 10^{-5} \mathrm{~m} / \mathrm{m} \mathrm{K}$ ). It is assumed that the heat expansion effect will even out with respect to the trend estimates but will contribute to the observed noise as well as to the periodical components, though mainly on the z-coordinate. The contribution to the periodical components may be both to the seasonal variations as well as to the diurnal variations. The latter will appear as noise in the measurements due to the short wavelengths with respect to the sampling interval. As the amplitudes for the 
periodic components detected for the $\mathrm{z}$-coordinates for some of the control points are in the order of magnitude $2 \mathrm{~mm}$, they are likely influenced by the heat expansion of the pillars.

The time series used in this investigation were not fully ideal for spectral analysis, as there were some violations of the conditions of the Fourier analysis such as offsets, blunders and variable noise levels as well as missing observations which had to be corrected. Among others, this required interpolation to get equidistant data. This interpolation may indeed influence the results from the spectral analysis. In order to get an idea of how the results were affected, various interpolations methods such as linear, nearest neighbour and cubic splines were tested. However, the choice of interpolation method seemed to only have minor influences on the resulting periods. Also, imperfections in the trend model may affect the outcome from spectral analysis. Parameter estimates will generally be affected depending on whether all parameters are estimated simultaneously or the trend part is estimated separately i.e. before the periodical components are estimated. Some limited testing indicated, however, that this effect is relatively small. Therefore the estimation was carried out in a two step manner by first removing the linear trends before the periodical components were estimated from the resulting residuals. Moreover, inserting zero-residuals within the gaps probably caused some reduction of the estimated amplitudes of the periodic components.

All extensometers show high correlation with the groundwater level (Table 4, Fig. 12). However, the behaviour of Ext A and Ext B are probably affected by some fractures in the vicinity of these extensometers. Therefore, Ext $\mathrm{C}$ is regarded as the most representative for the upper part of the slope. Correlations for the other GPS control points were low (Table 4) and therefore considered as neglectable from a practical point of view.

It is already established that there are certain periodic variations in displacement rates at the Åknes rockslide due to seasonal variations in precipitation and temperature. During spring and autumn the displacement rates are larger due to snowmelt and increased precipitation, respectively (Grøneng et al., 2009). However, statistical evaluations carried out in this analysis provide a formalized means for quantifying these relations which may be useful both for interpretation and prediction of future displacements.

Even though some of the time series, particularly those from extensometers, can be well described by higher order polynomials, it is advantageous to use a model based on linear trends combined with periodical components. With respect to prediction of future displacements, the performance of a model based on higher order polynomials may be completely unsatisfactory outside the observation interval. Moreover, interpreting estimated model parameters from linear trend models with periodic components are more straightforward than for higher order polynomial models.

On the basis of the analysis reported in this paper some recommendations regarding design of future displacement measurements can be given; the continuous measurements are preferably to the yearly survey campaigns. This is evident from the currently available GPS time series. If the displacement vectors were derived from the difference in coordinate values, from the mean of the initial part of the time series to the mean of the end of the same time series, this would lead to erroneous results compare to the displacement rates derived from trend estimates. This is a result of the offsets within the time series (Fig. 8). Similarly, the contradictions between vertical displacements, in the south-eastern part of the slope, for the period 2005-2006 (Figs. 6, 7) were most likely caused by a systematic error (vertical shift) related to the GPS measurements carried out in 2006.

Moreover, a larger number of control points and better spatial coverage of the unstable area is preferable with respect to future analyses. More control points located at the assumed stable parts of the slope may also be beneficial. As there may be un-modelled effects related to the GPS measurements, more control points located at the stable parts of the slope would help identifying such un-modelled effects as well as being a validation of the estimated accuracy. However, in most cases there will by practical limitations regarding the number and locations of the control points. For the Åknes case, some parts of the slope are exposed to frequent snow avalanches whereas others have limited GPS satellite coverage which makes those areas less suitable.

\section{Summary and conclusions}

A statistical approach for analyzing various types of surface displacement data is presented. Different displacement datasets, including data from survey campaigns, carried out with GPS and total stations, as well as time series from continuously monitored GPS and extensometers control points, were analysed. These datasets were collected at the Åknes site in western Norway, one of the world's most investigated rockslides.

The main focus for this investigation was time series analysis. Results from time series analyses show that displacements at Åknes can be modelled as linear trends superimposed with periodic components. This means that there are periodical fluctuations but no persistent acceleration.

The annual displacement rates, estimated from GPS and extensometer time series, range from a few millimetres to about $8 \mathrm{~cm}$, whereas the periodical fluctuations typically have maximum amplitudes of $1-2 \mathrm{~mm}$. Some of the estimated periodic components show annual or biannual variations which are most likely caused by changes in groundwater level, precipitation and snowmelt.

High correlations between displacements and the groundwater level, measured in a borehole at the upper part of the slope, were evident for extensometers located across the back scarp. However, for the GPS control points located further 
down the slope, the correlations were low and therefore considered as neglectable from a practical point of view.

The three most similar displacement vectors, derived from GPS time series, were formally tested for equality by means of hypothesis testing. The hypothesis regarding equality was rejected. This supports the complex displacement pattern evident from previous GPS and total station survey campaigns as well as the assumption of a number of individual moving blocks.

Some data sets, particularly the GPS time series, were not fully ideal in terms of missing observations, offsets and gross errors. These problems were resolved by various techniques such as gross error removal, interpolation and offset estimation. However, such flaws in data make additional contributions to the uncertainties associated with the estimated displacements.

We believe that the statistical approach reported in this paper can be useful to other landslide assessments as well as for future analyses of the Åknes rockslide.

Acknowledgement. Thanks to T. Eiken, University of Oslo, for providing results from LS adjustment of GPS and total station survey campaigns. We also thank H. Nahavandchi, Norwegian University of Science and Technology and T. A. Haakonsen, Trondheim municipality, for valuable comments and suggestions regarding spectral analysis. L. H. Blikra and G. Grøneng, Norwegian University of Science and Technology, are acknowledged for their useful comments regarding geological aspects.

Edited by: T. Glade

Reviewed by: J. Dehls and another anonymous Referee

\section{References}

Caspary, W. F., Haen, W., and Borutta, H.: Deformation analysis by statistical methods, Technometrics, 32, 49-57, 1990.

Brückl, E., Brunner, F. K., and Kraus, K.: Kinematics of a deep seated landslide derived from photogrammetric, GPS and geophysical data, Eng. Geol., 88, 149-159, 2006.

Cooper, M. A. R.: Control surveys in civil engineering, Collins Publisher, London, UK, 1987.

Davis, J. C.: Statistics and Data Analysis in Geology, 3rd ed., John Wiley \& Sons, USA, 2002.

Denli, H. H. and Deniz, R.: Global Congruency Test Methods for GPS Networks, J. Surv. Eng., 129, 95-98, 2003.

Ganerød, G. V., Grøneng, G., Rønning, J. S., Dalsegg, E., Elvebakk, H., Tønnesen, J. F., Kveldsvik, V., Eiken, T., Blikra, L. H., and Braathen, A.: Geological Model of the Åknes Rockslide, western Norway, Eng. Geol., 102, 1-18, 2008.
Grøneng, G., Christiansen, H. H., and Blikra, L. H.: Meteorological Control on Seasonal Rock Deformation in the Åknes Sliding Area, Western Norway, in preparation, 2009.

Haberler-Weber, M.: Analysis and interpretation of geodetic landslide monitoring data based on fuzzy systems, Nat. Hazard. Earth Sys., 5, 755-760, 2005.

Johnson, R. A. and Wichern, D. W.: Applied Multivariate Statistical Analysis, 5th ed., Prentice Hall, New Jersey, USA, 2002.

Kennie, T. J. M. and Petrie, G.: Engineering surveying technology, Thomson Litho Ltd, Scotland, 1990.

Koch, K. R.: Parameter estimation and hypothesis testing in linear models, 2nd ed., Springer-Verlag, Germany, 1999.

Kveldsvik, V., Eiken, T., Ganerød, G. V., Grøneng, G., and Ragvin, N.: Evaluation of movement data and ground conditions for the Åknes rock slide, The International Symposium on Stability of Rock Slopes, The African Institute of Mining and Metallurgy, 279-299, 2006.

Miller, A. J.: Subset selection in regression, 2nd ed., Chapmann \& Hall/CRC, 2002.

Montgomery, D. C., Peck, E. A., and Vining, G. G.: Introduction to linear regression analysis, 3rd ed. John Wiley \& Sons, USA, 2001.

Nordvik, T., Grøneng, G., Ganerød, G. V., Nilsen, B., Harding, C., and Blikra, L. H.: Geovisualization, geometric modelling and volume estimation of the Ånes rockslide, western Norway, B. Eng. Geol. Environ., 68(2), 245-256, doi:10.1007/s10064-0090198-x, 2009.

Petly, D. N., Bulmer, M. H., and Murphy, W.: Patterns of movement in rotational and translational landslides, Geology, 30, 719-722, 2002.

Pytharouli, S. I., Kontogianni, V. A., and Stiros, S. C.: Kinematics of two deep-seated landslides in Greece, Geotechnical Engineering, 160, 179-183, 2007.

Savšsek-Safiæ, S., Ambrožiè, T., Stopar, B., and Turk, G.: Determination of Point Displacements in the Geodetic Network, J. Surv. Eng.-ASCE, 132, 58-63, 2006.

Sitros, S. C., Vichas, C., and Skourtis, C.: Landslide Monitoring based on geodetically derived distance changes, J. Surv. Eng.ASCE, 130, 156-162, 2004.

Teza, G., Pesci, A., Genevois, R., and Galgaro, A.: Characterization of landslide ground surface kinematics from terrestrial laser scanning and strain field computation, Geomorphology, 97, 424437, 2008.

Tzenkov, T. and Gospodinov, S.: Geometric Analysis of Geodetic Data for Investigation of 3D Landslide Deformations, Natural Hazard Review, 4, 78-81, 2003.

Wei, W. W. S.: Time Series Analysis, Addison-Wesley Publishing Company, USA, 1990. 